\title{
p53 siRNA inhibits apoptosis of U2OS cells treated with azurin
}

\author{
XIAOBO YAN ${ }^{1}$, JIE FENG $^{2}$, ZHAOMING YE $^{1}$, XUDONG MIAO $^{1}$, WEIXU LI $^{1}$ and DISHENG YANG ${ }^{1}$ \\ ${ }^{1}$ Department of Orthopaedics, 2nd Affiliated Hospital, School of Medicine, Zhejiang University; ${ }^{2}$ Institute \\ for Orthopaedic Research, Zhejiang University, Hangzhou, Zhejiang 310058, P.R. China
}

Received February 25, 2011; Accepted August 1,2011

DOI: $10.3892 / \mathrm{mmr} .2011 .544$

\begin{abstract}
The bacterial redox protein azurin selectively induces apoptosis in human osteosarcoma U2OS cells. We constructed a p53 siRNA to test the role that p53 plays in the apoptosis-inducing role of azurin in U2OS cells. Cells treated with p53 siRNA and azurin showed more viable cells, a lower apoptosis rate, lower caspase-3 activity, and up-regulation of bcl-2, downregulation of bax compared to cells treated with negative siRNA and azurin. Cells treated with negative siRNA and azurin yielded positive TUNEL dying, whereas cells treated with p53 siRNA and azurin yielded few positive cells. These results suggested that p53 siRNA was capable of inhibiting the apoptosis induced by azurin 2 days after treatment with p53 siRNA and azurin. Azurin may induce apoptosis through combination with $\mathrm{p} 53$. The decrease in $\mathrm{p} 53$ protein levels did not inhibit cell apoptosis rates, but rather increased these rates in U2OS osteosarcoma cells 3 days after treatment with p53 siRNA. Since U2OS cells are p53 wild-type cancer cells, p53 potentially acts as an oncogene in U2OS osteosarcoma cells. Treatment with azurin may transform the function of p53 from inhibiting to inducing apoptosis.
\end{abstract}

\section{Introduction}

Azurin is a copper-containing protein involved in electron transfer during denitrification (1). Previously, purified azurin was found to exhibit cytotoxicity to osteosarcoma U2OS cells. Azurin selectively induced apoptosis in U2OS cells. The induction of apoptosis was closely associated with the downregulation of bcl-2, upregulation of bax, and activation of caspase-3 (2). In that study, we also constructed a eukaryotic expression plasmid containing the azurin gene with an influenza virus hemagglutinin 9 peptide HA epitope tag and transfected the recombinant plasmid pcDNA3.1(+)/azurin into U2OS cells. Transfection of the recombinant plasmid pcDNA3.1(+)/azurin significantly induces apoptosis in U2OS cells. This is closely associated with the upregulation of the

Correspondence to: Dr Weixu Li, Department of Orthopaedics, 2nd Affiliated Hospital, School of Medicine, Zhejiang University, 88 Jie Fang Road, Hangzhou, Zhejiang 310009, P.R. China

E-mail: zrgk5@163.com

Key words: p53, siRNA, azurin, transfection transcriptional level of the bax and p53 genes and the downregulation of that of the bcl-2 gene (3). The tumor-suppressor protein p53 is involved in the regulation of cell growth, genomic stability and cell death. Previous studies suggested that azurin from Pseudomonas aeruginosa is the only bacterial protein that is capable of entering cancer cells and interacting with p53 to promote cell death (4). Molecular interaction between the p53 tumor suppressor and the copper protein azurin (AZ) has been shown to enhance p53 stability, and thus anti-tumor function, opening new perspectives in cancer treatment (5). In the present study, p53 siRNA was used to test the function of p53 in the apoptosis-inducing role of azurin.

\section{Materials and methods}

Materials. Azurin purchased from Sigma (St. Louis, MO, USA) was dissolved in RPMI-1640 or Eagle's minimal essential medium (MEM) to a final concentration of $1 \mathrm{mg} / \mathrm{l}$ and stored in a dark-colored bottle at $4^{\circ} \mathrm{C}$ as stock solution. The stock was diluted to the required concentration immediately prior to use with growth media. Antibodies against bcl-2, bax, p53 and actin were purchased from Santa Cruz Biotechnology (Santa Cruz, CA, USA). The annexin V-FITC kit was purchased from BD Biosciences (San Jose, CA, USA). The terminal deoxynucleotidyl transferase-mediated dUTP nick-end labeling (TUNEL) cell apoptosis detection kit and caspase-3 activity assay kit were provided by the Beyotime Institute of Biotechnology (Haimen, China). p53 siRNA was synthesized by Shanghai GenePharma Co., Ltd. The sense primer was 5'-CUACUUCCUGAAAACAACGdTdT-3', and the anti-sense was 5'-CGUUGUUUUCAGGAAGUAGdTdT-3'.

Cell line and culture conditions. U2OS, a human osteosarcoma cell line, was purchased from the American Type Culture Collection (ATCC, Rockville, MD, USA). The cells were cultured in RPMI-1640 with $10 \%$ heat-inactivated fetal bovine serum (FBS), $2 \mathrm{mM}$ L-glutamine, $100 \mathrm{U} / \mathrm{ml}$ penicillin, and $100 \mu \mathrm{g} / \mathrm{ml}$ streptomycin at $37^{\circ} \mathrm{C}$ in a humidified atmosphere containing $5 \% \mathrm{CO}_{2}$.

Transfection. Transfection of the U2OS cells was carried out in 6-well plates according to the instructions for Lipofectamine $^{\mathrm{TM}}$ 2000. A total of $4 \mu \mathrm{g}$ of p53 siRNA and the negative control siRNA were transfected separately into U2OS cells. 
MTT cell growth assay. The cytotoxic effects of azurin on U2OS cells were assessed by MTT assay, which is based on the reduction of MTT by the mitochondrial dehydrogenase of intact cells to a purple formazan product. The cells were suspended in a 96-well plate (Costar; Corning, NY, USA) at a density of $2 \times 10^{4}$ cells per well. After azurin treatment, the medium containing azurin was carefully removed by aspiration. A total of $20 \mu \mathrm{l}$ of MTT solution $\left(5 \times 10^{3} \mathrm{mg} / \mathrm{l}\right)$ was added to each well $4 \mathrm{~h}$ before the end of incubation. The supernatant was removed and $150 \mu \mathrm{l}$ DMSO was added to each well. An ELISA reader was used to measure the absorbance at $490 \mathrm{~nm}$.

Caspase-3 activity assay. Caspase- 3 activity was measured by cleavage of chromogenic caspase substrates, Ac-DEVD-pNA (acetyl-Asp-Glu-Val-Asp p-nitroanilide) and Ac-LEHD-pNA (acetyl-Leu-Glu-His-Asp p-nitroanilide). Approximately $50 \mu \mathrm{g}$ of total protein was added to the reaction buffer containing Ac-DEVD-pNA ( $2 \mathrm{mM})$ or Ac-LEHD-pNA $(2 \mathrm{mM})$ and incubated for $2 \mathrm{~h}$ at $37^{\circ} \mathrm{C}$. The absorbance of yellow pNA cleaved from its corresponding precursors was measured using a spectrometer at $405 \mathrm{~nm}$. The specific caspase activity, normalized for total proteins of cell lysates, was then expressed as a fold of the baseline caspase activity of the control cells cultured in DMEM with $10 \%$ FBS.

TUNEL. The TUNEL method was performed to label the 3 '-end of fragmented DNA of the apoptotic pulmonary arterial smooth muscle cells (PASMCs). The cells treated as indicated were fixed with $4 \%$ paraformaldehyde/phosphatebuffered saline (PBS), rinsed with PBS, then permeabilized by $0.1 \%$ Triton X-100 for biotin-TUNEL the fragmented DNA of the apoptotic cells. Biotin was then conjugated with streptavidin-horseradish peroxidase (HRP) and colored with DAB using a TUNEL cell apoptosis detection kit. The biotin-labeled TUNEL-positive cells were observed under a microscope.

Apoptosis and cell-cycle analysis were quantified by fluorescence-activated cell sorting (FACS). Determination of apoptosis and secondary necrosis utilizes the high affinity of annexin $\mathrm{V}$ to phosphatidylserine, which is exposed on the surface of apoptotic cells. U2OS cells $\left(1 \times 10^{6}\right)$ were trypsinized to detach any adherent cells. Cells were harvested and washed in PBS, resuspended in binding buffer $(10 \mathrm{mmol} / \mathrm{l} \mathrm{HEPES} /$ $\mathrm{NaOH}, 140 \mathrm{mmol} / \mathrm{l} \mathrm{NaCl}, 2.5 \mathrm{mmol} / \mathrm{l} \mathrm{CaCl}, \mathrm{pH} 7.4$ ), and incubated with $0.25 \mathrm{mg} / \mathrm{ml}$ fluorescein isothiocyanate (FITC)conjugated annexin $\mathrm{V}$ and $10 \mathrm{mg} / \mathrm{ml}$ propidium iodide (PI). The mixture was kept on ice for $5 \mathrm{~min}$ and the cell fluorescence was measured by two-parameter flow cytometry (FACS Calibur; Becton-Dickinson, San Jose, CA, USA). When green fluorescence (FITC) was plotted against red fluorescence (PI), three distinct cell populations were detected in a dotplot: viable cells (FITC-/PI-), apoptotic cells (FITC+/PI-), and secondary necrotic cells (FITC+/PI+). A minimum of 10,000 events was counted per sample and data were reported as the percentage of apoptotic cells (annexin V-FITC+/PI-). Cell-cycle analysis was also evaluated with FACS using a cell-cycle analysis kit (Cycle Test Plus, Becton-Dickinson). Cell cycle distribution was based on $2 \mathrm{~N}$ and $4 \mathrm{~N}$ DNA content for DNA content analysis using BD CellQuest software v 6.0. as previously described.
Western blot analysis. Cells were lysed by $200 \mu 1$ M-PER ${ }^{\circledR}$ reagent (Pierce Biotechnology, Rockford, IL, USA). Centrifugation was carried out at $14,000 \mathrm{x}$ g for $10 \mathrm{~min}$ at $0^{\circ} \mathrm{C}$. The supernatants were then collected, and the proteins were separated on $12 \%$ SDS-PAGE. After electrophoresis, the protein blots were transferred to a nitrocellulose membrane. The membrane was blocked with 5\% non-fat milk in TBST and incubated overnight with polyclonal anti-HA tag antibody at $37^{\circ} \mathrm{C}$ for $1 \mathrm{~h}$. After 3 washes with TBST, the membrane was incubated at $37^{\circ} \mathrm{C}$ for $1 \mathrm{~h}$ with HRP-conjugated secondary antibody diluted with TBST (1:500). The detected protein signals were visualized by an enhanced chemilluminescence detection system (Amersham, Arlington Heights, IL, USA).

Statistical analysis. Data are presented as the mean \pm SD of 3 replicates from 4 separate experiments. Collected data are expressed as the mean $\pm \mathrm{SD}$. Statistical analysis was performed by one-way ANOVA to determine the difference within groups.

\section{Results}

MTT of cells treated with p53 siRNA and azurin. U2OS cells were transfected with p53 siRNA and negative control siRNA separately. Usually, siRNA takes 4-6 h to enter cells. Azurin was added $6 \mathrm{~h}$ after siRNA. MTT assays were performed after $48 \mathrm{~h}$ since azurin shows the greatest effects at this time point. The optical density (OD) value is the number of viable cells. Azurin was found to decrease the viability of cells treated with negative control siRNA $(\mathrm{P}<0.05) 2$ days after the beginning of azurin treatment. The viability of cells treated with p53 siRNA was found to be higher than that of cells treated with p53 siRNA and azurin $(\mathrm{P}<0.05)$. No significant difference was found between cells treated with control siRNA and those treated with p53 siRNA after 2 days $(\mathrm{P}>0.05)$, but this was not the case after 3 days $(\mathrm{P}<0.05)$. In addition, after 3 days cells treated with p53 siRNA and azurin showed lower levels of cell viability compared to cells treated with control siRNA + azurin and cells treated with p53 siRNA alone $(\mathrm{P}<0.05)$.

Effects of p53 siRNA on morphology of U2OS cells treated with azurin. Under the microscope, cells treated with control siRNA showed normal morphology. The plate was full of living cells after 2 days of growth. The group treated with control siRNA and azurin showed few viable cells with numerous round, black granules, which consisted of dead or apoptotic cells starting to float. The group treated with p53 siRNA and azurin had the most viable cells with normal cell morphology; however, numerous round, black granules were still evident.

Effects of p53 siRNA on apoptosis in U2OS cells treated with azurin. U2OS cell apoptosis was measured in a FACS. Annexin V-FITC+/PI- exhibited apoptotic cells. Following treatment with p53 siRNA and control siRNA for 4-6 h, azurin was added. In cells treated with control siRNA and azurin, an apoptotic peak was observed and the rate of apoptosis was $11.26 \%$. In cells treated with p53 siRNA and azurin, the rate of apoptosis was $7.73 \%$. Cells treated with negative siRNA showed an apoptotic rate of $3.02 \%$. 


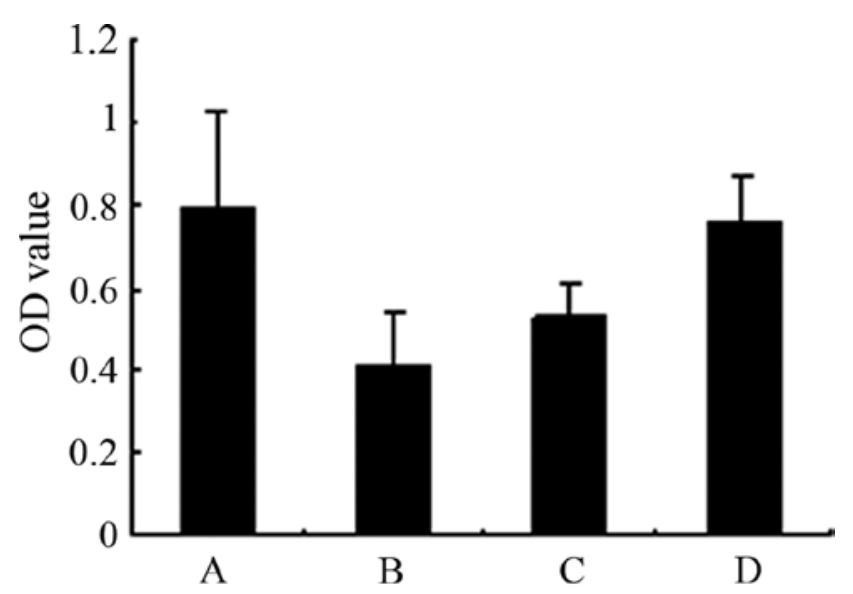

Figure 1. Viability of cells treated with p53 siRNA and azurin. Cells were treated with p53 siRNA and control siRNA for $6 \mathrm{~h}$. Azurin was then added. Cell viability was measured by a MTT assay 2 days later. (A) Cells treated with control siRNA. (B) Cells treated with control siRNA and azurin. (C) Cells treated with p53 siRNA and azurin. (D) Cells treated with p53 siRNA.

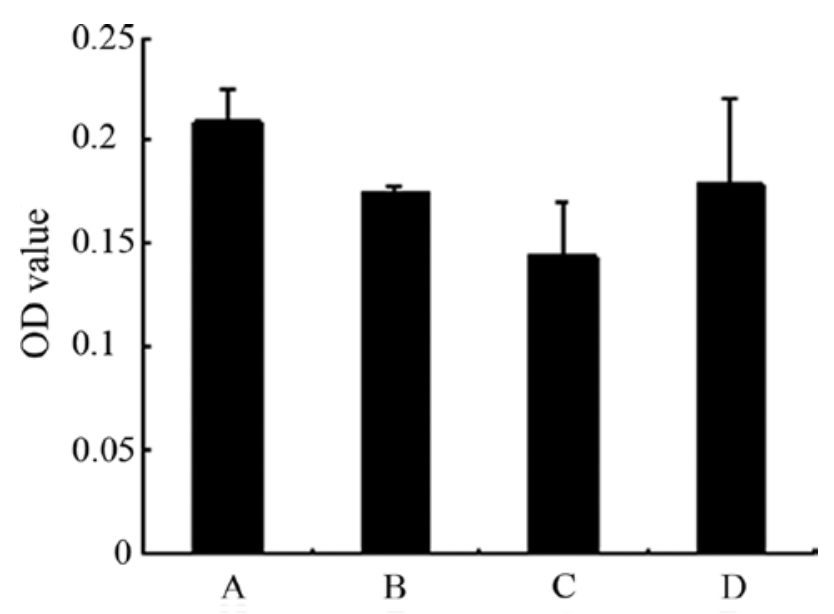

Figure 2. Viability of cells treated with p53 siRNA and azurin. Cells were treated with p53 siRNA and control siRNA for $6 \mathrm{~h}$. Azurin was then added. Cell viability was measured by a MTT assay 3 days later. (A) Cells treated with control siRNA. (B) Cells treated with control siRNA and azurin. (C) Cells treated with p53 siRNA and azurin. (D) Cells treated with p53 siRNA.
A

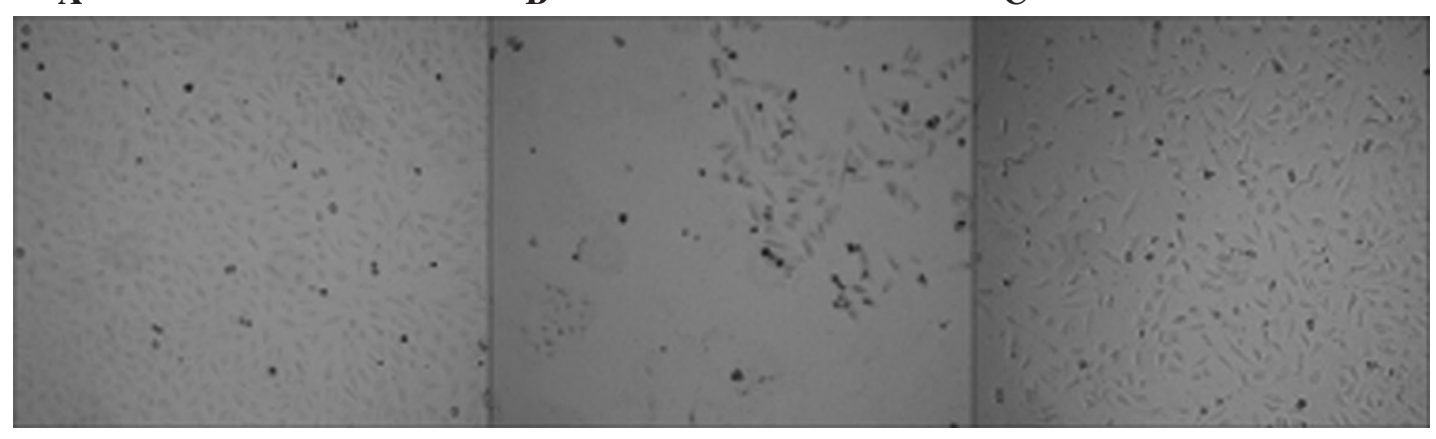

Figure 3. Morphology of U2OS cells treated with p53 siRNA and azurin. Cells were treated with p53 siRNA and control siRNA for 6 h. Azurin was then added to the final concentration of $50 \mu \mathrm{g} / \mathrm{ml}$. Cells were observed under a microscope 2 days later. (A) Cells treated with control siRNA. (B) Cells treated with control siRNA and azurin. (C) Cells treated with p53 siRNA and azurin.

Effects of p53 siRNA on TUNEL tests of U2OS cells treated with azurin. Apoptotic cells show nuclear fragmentation that was end-labeled by biotin-dUTP and colored by DAB. Most cells treated with control siRNA and azurin showed deep coloration of the nucleus. A few cells treated with p53 siRNA and azurin also showed colored nuclei.

Effects of p53 siRNA on protein expression of U2OS cells treated with azurin. After $48 \mathrm{~h}$, Western blot analysis showed that p53 siRNA had an obvious effect on the bcl-2 and bax protein levels in cells treated with azurin. The expression of bcl-2 protein increased in U2OS cells treated with p53 siRNA and azurin compared to cells treated with negative siRNA and azurin, whereas that of bax proteins was reduced, suggesting that $\mathrm{p} 53$ siRNA inhibited azurin-induced apoptosis through the downregulation of bax and upregulation of bcl-2. p53 expression in cells treated with p53 siRNA demonstrated that p53 siRNA actually interfered with the expression of p53.

Effects of p53 siRNA on caspase-3 activity of U2OS cells treated with azurin. Caspase-3 activity was evaluated by
Beyotime caspase- 3 activity assay kit. The caspase- 3 activity of cells treated with control siRNA and azurin was higher than that of cells treated with control siRNA only $(\mathrm{P}<0.05)$, demonstrating the apoptosis-inducing effect of azurin. The caspase- 3 activity of cells treated with p53 siRNA and azurin was lower than that of cells treated with control siRNA and azurin $(\mathrm{P}<0.05)$, demonstrating the inhibitory effect of p53 on azurin.

\section{Discussion}

p53 is a human tumor suppressor that regulates a number of cellular processes such as cell growth, genomic stability, and cell death. Studies have demonstrated that the bacterial redox protein azurin is capable of entering cancer cells and inducing apoptosis through p53 stabilization, resulting in a regression of tumor growth (6). The tumor suppressor p53 interacts with the redox copper protein azurin forming a complex that is crucial to azurin-induced apoptosis (7). The half-life of the tumor suppressor p53 can be increased by interaction with the bacterial protein azurin, resulting in enhanced anti-tumor 
A

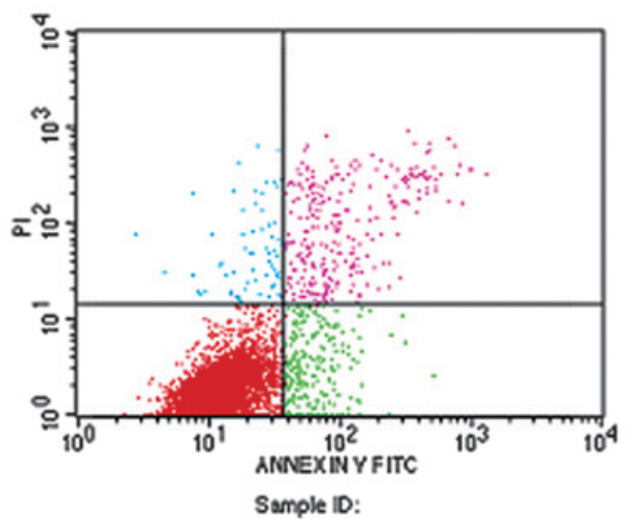

Qued 8 Gered

U $\quad 0.84$

UR $\quad 3.24$

LL $\quad 92.90$

LR 3.02
B

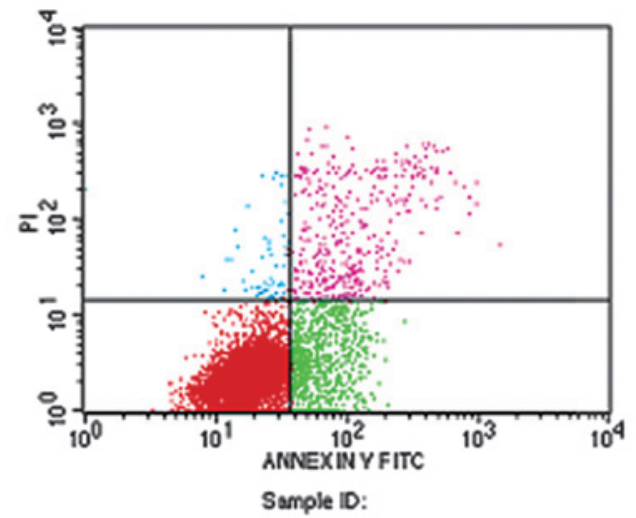

C
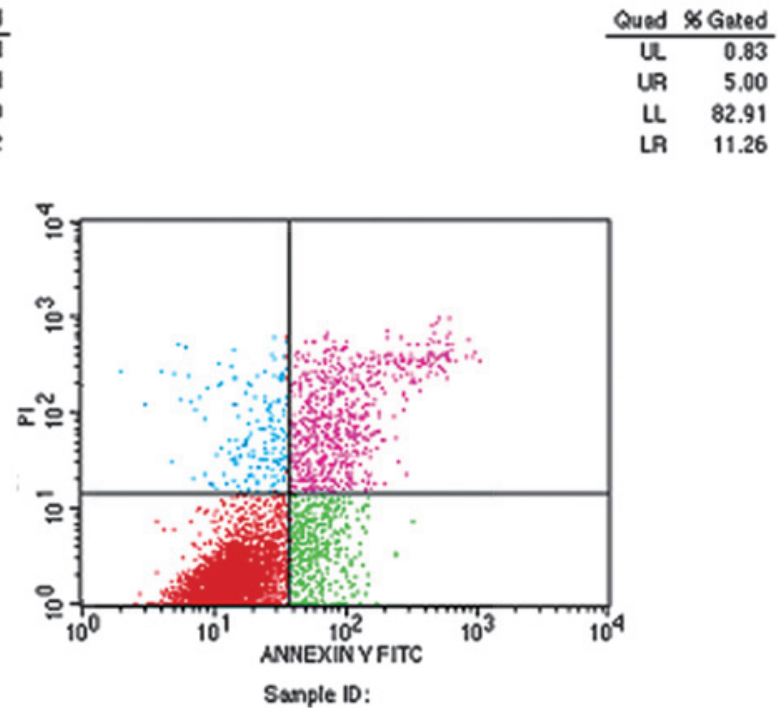

\begin{tabular}{cr} 
Quad & \% Gated \\
\hline UL & 2.88 \\
UR & 11.80 \\
LL & 77.58 \\
LR & 7.73
\end{tabular}

Figure 4. Apoptosis of U2OS cells treated with p53 siRNA and azurin. Cells were treated with p53 siRNA and control siRNA for 6 h. Azurin was then added to the final concentration of $50 \mu \mathrm{g} / \mathrm{ml}$. Cells were tested by flow cytometry 2 days later. (A) Cells treated with control siRNA. (B) Cells treated with control siRNA and azurin. (C) Cells treated with p53 siRNA and azurin.

A B

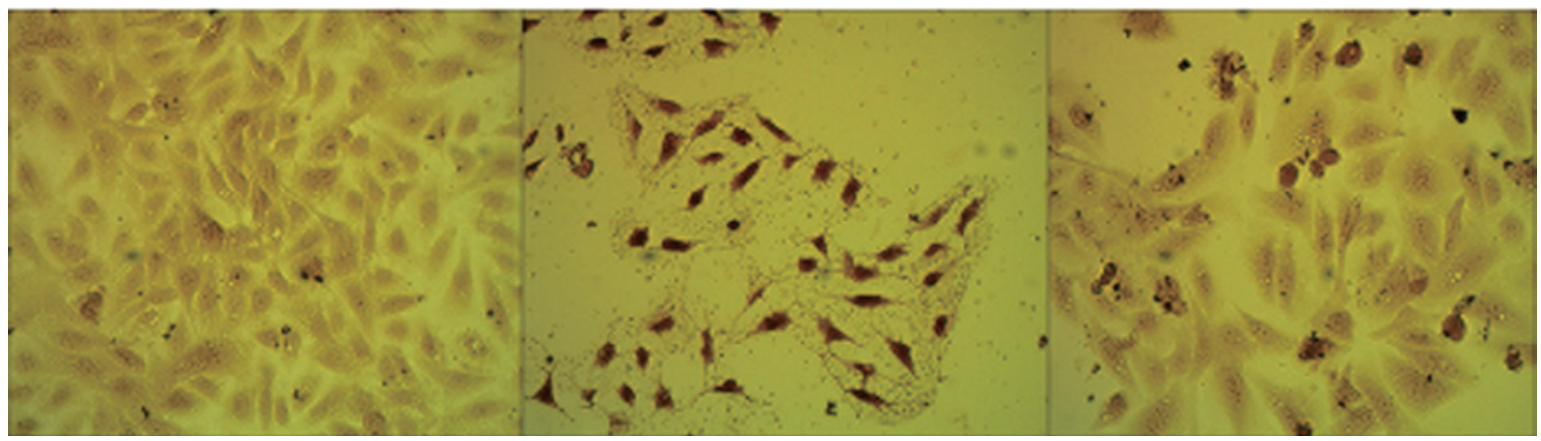

Figure 5. TUNEL test of U2OS cells treated with p53 siRNA and azurin. Cells were treated with p53 siRNA and control siRNA for 6 h. Azurin was then added to the final concentration of $50 \mu \mathrm{g} / \mathrm{ml}$. Apoptotic cells were labeled using a TUNEL cell apoptosis detection kit 2 days later. (A) Cells treated with control siRNA. (B) Cells treated with control siRNA and azurin. (C) Cells treated with p53 siRNA and azurin.

activity. An understanding of the molecular mechanisms on the basis of this phenomenon can facilitate new anti-cancer strategies (8).
RNA interference is the process of sequence-specific, transcriptional/post-transcriptional gene silencing through siRNA. RNA interference is a popular method of regulating gene 

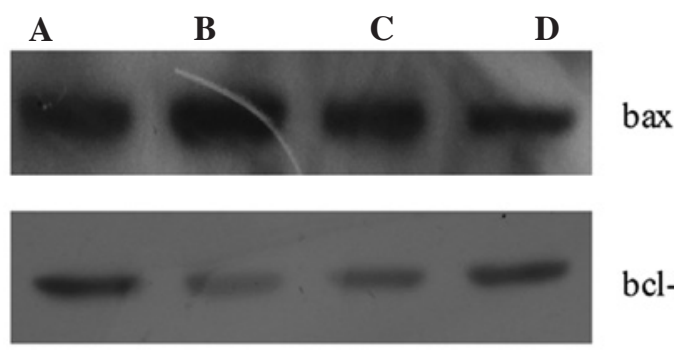

bcl-2

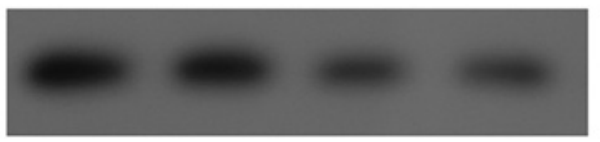

p53

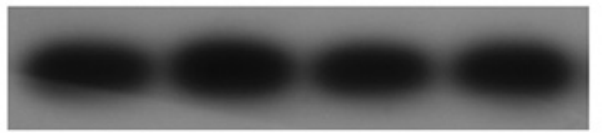

$\beta$-actin

Figure 6. Bcl-2, bax and p53 expression in U2OS cells treated with p53 siRNA and azurin. Cells were treated with p53 siRNA and control siRNA for $6 \mathrm{~h}$. Azurin was then added to the final concentration of $50 \mu \mathrm{g} / \mathrm{ml}$. Cell proteins were extracted and separated by SDS-PAGE electrophoresis 2 days later. (A) Cells treated with control siRNA. (B) Cells treated with control siRNA and azurin. (C) Cells treated with p53 siRNA and azurin. (D) Cells treated with p53 siRNA.

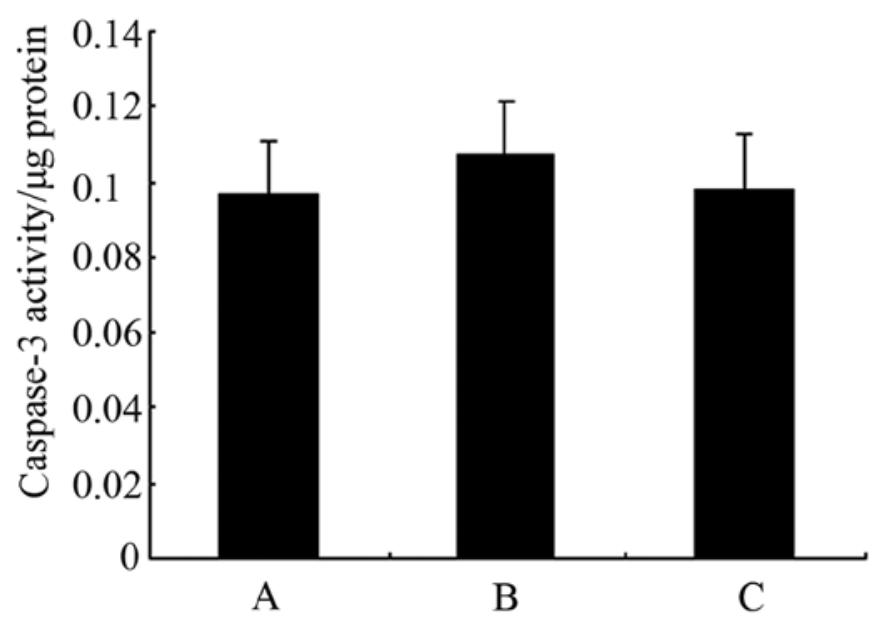

Figure 7. Caspase activity of U2OS cells treated with p 53 siRNA and azurin. Cells were treated with p53 siRNA and control siRNA for $6 \mathrm{~h}$. Azurin was then added to the final concentration of $50 \mu \mathrm{g} / \mathrm{ml}$. Caspase- 3 activity was evaluated 2 days later. (A) Cells treated with control siRNA. (B) Cells treated with control siRNA and azurin. (C) Cells treated with p53 siRNA and azurin.

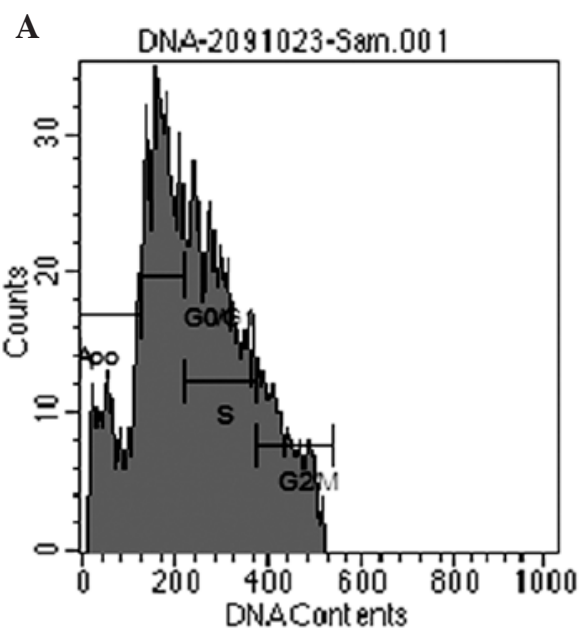

C

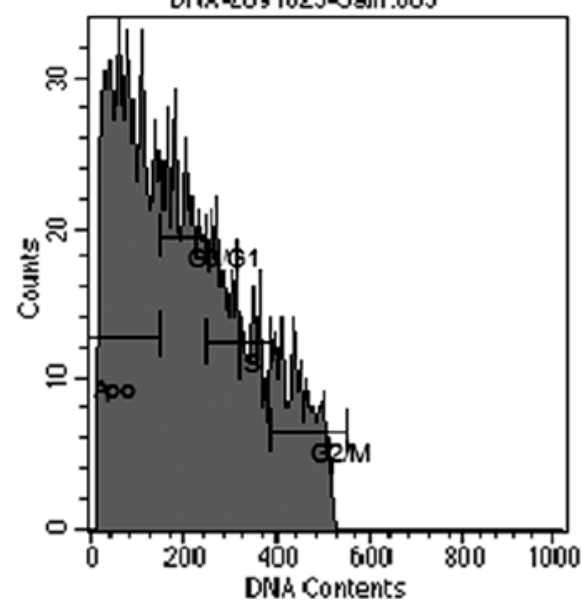

B

Fik: DNA-2091023-Sam.001

\begin{tabular}{rrr} 
Mrker & \% Goted & Mean \\
\hline All & 100.00 & 241.49 \\
Ap & 13.48 & 81.25 \\
GQ/G1 & 33.55 & 173.88 \\
S & 40.55 & 288.37 \\
G2M & 13.48 & 430.52
\end{tabular}

File: DNA.2091023-Sam.002

\begin{tabular}{rrr} 
Mrker & \% Grted & Mean \\
\hline Al & 100.00 & 225.16 \\
ADO & 32.20 & 82.04 \\
GO/G1 & 28.61 & 195.41 \\
S & 24.78 & 313.84 \\
G2M & 14.88 & 443.97
\end{tabular}

D

Fik: DNA-2091023-Sam.003

\begin{tabular}{rrr} 
Marker & \% Gated & Megn \\
\hline All & 100.00 & 202.58 \\
ADO & 42.22 & 81.37 \\
GO/G1 & 24.85 & 199.34 \\
S & 21.99 & 311.72 \\
G2M & 11.83 & 445.54
\end{tabular}

FiE: DNA-2091023-Sam.004

\begin{tabular}{rrr} 
Marker & \% Gsted & Mean \\
\hline Al & 100.00 & 128.33 \\
ApD & 68.08 & 81.87 \\
GQ/G1 & 26.24 & 190.57 \\
S & 6.98 & 288.08 \\
G2M & 1.25 & 409.55
\end{tabular}
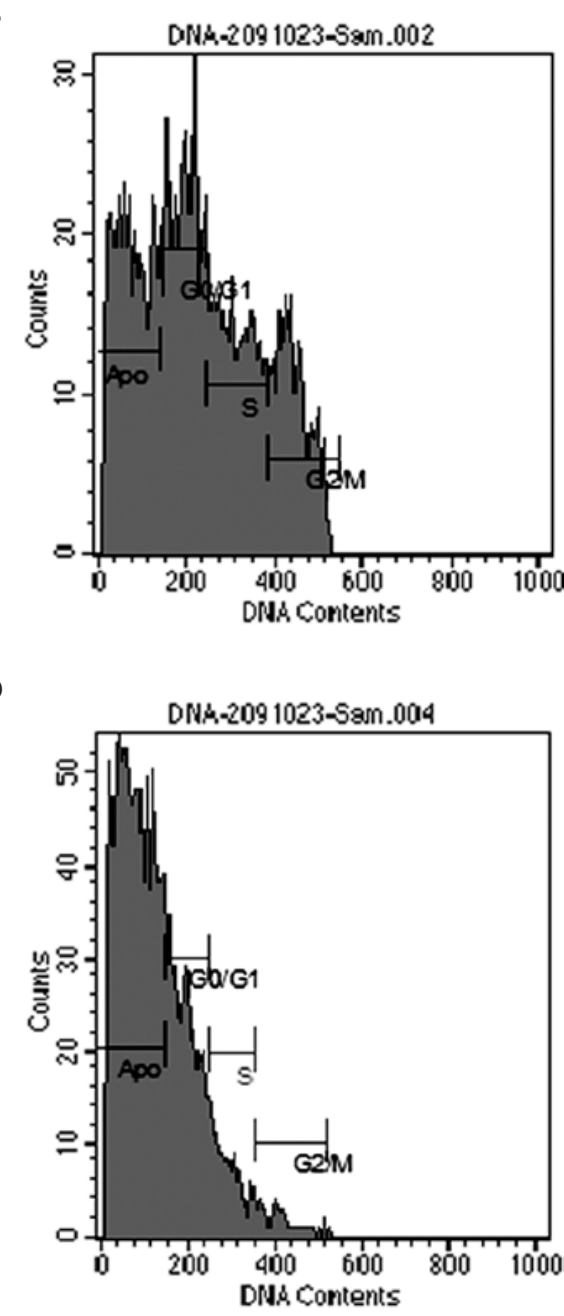

Figure 8. Apoptosis in cells treated with p53 siRNA and azurin. Cells were treated with p53 siRNA and control siRNA for 6 h. Azurin was then added. Cell apoptosis was measured by flow cytometry 3 days later. (A) Untreated control cells. (B) Cells treated with control siRNA. (C) Cells treated with control siRNA and azurin. (D) Cells treated with p53 siRNA and azurin. 
expression. In the present study, we constructed p53 siRNA that would interfere with p53 expression. p53 siRNA combines with p53 mRNA and inhibits its translation, reducing p53 protein levels. In this study, cells treated with p53 siRNA and azurin showed more living cells, a lower rate of apoptosis, lower caspase-3 activity, upregulation of bcl-2, and downregulation of bax compared to cells treated with negative siRNA and azurin. Cells treated with negative siRNA and azurin exhibited positive TUNEL death, whereas cells treated with p53 siRNA and azurin exhibited few positive cells.

Bax is a crucial mediator and the loss of this pro-apoptotic protein contributes to drug resistance in human cancers (9). As a tumor suppressor, Bax mediates p53-induced apoptosis and increases sensitivity to chemotherapy-induced apoptosis $(10,11)$. Bcl-2 is an anti-apoptotic protein. When it is activated or prevalent, apoptosis is inhibited. The abnormal overexpression of bcl-2 has frequently been observed in various types of human cancer. It has been suggested that p53 siRNA is capable of inhibiting azurin-induced apoptosis. Azurin may induce apoptosis through the combination with $\mathrm{p} 53$.

Azurin is not capable of inducing apoptosis without the p53 protein, if azurin's effect is p53-dependent. However, the tumor suppressor protein $\mathrm{p} 53$ is known to either induce apoptosis or arrest growth depending on cellular background (12). p53 siRNA decreases p53 protein levels, and this decrease in 553 is capable of inhibiting cancer cell apoptosis. When p53 siRNA was transfected into U2OS cells, it increased the viability of cells treated with azurin over that of cells treated with negative siRNA and azurin. This increase was caused by the decrease in $\mathrm{p} 53$ protein rather than by the decrease in the combination of azurin and p53 protein as follows: After 2 days of treatment, the viability of cells treated with negative siRNA and that of cells treated with p53 siRNA were identical, showing no significant difference (Fig. 2). The decrease in $\mathrm{p} 53$ protein caused by $\mathrm{p} 53$ siRNA suggested that no functional effect was evident. Three days later, however, the viability of cells treated with p53 siRNA was lower than that of cells treated with negative siRNA $(\mathrm{P}>0.05)$. This reduction suggested that the decrease in $\mathrm{p} 53$ protein did not cause cell survival but rather cell death. This may explain the reason for the rate of apoptosis in cells treated with p53 siRNA and azurin was $66.08 \%$, greater than that of cells treated with negative siRNA and azurin, $42.22 \%, 3$ days later (Fig. 8).

p53 siRNA decreased levels of p53 protein (Fig. 6), but the decrease did not have any functional effect 2 days after treatment. The functional effect was observed 3 days later. The decrease in p53 protein levels did not inhibit cell apoptosis, but rather promoted cell apoptosis. Since U2OS cells are p53 wild-type cancer cells, it is likely that p53 plays the role of oncogene in U2OS osteosarcoma cells. Azurin treatment may transform the function of p53 from inhibiting apoptosis to inducing apoptosis. In this study, cells treated with p53 siRNA and azurin were found, 2 days later, despite the fact that the p53 protein itself decreased in cell numbers. p53 siRNA inhibited azurin-induced apoptosis. Azurin may induce apoptosis through a combination with $\mathrm{p} 53$. The decrease in p53 protein levels did not inhibit cell apoptosis, but rather promoted cell apoptosis in U2OS osteosarcoma cells 3 days after treatment. Since U2OS cells are p53 wild-type cancer cells, p53 plays a potential role as an oncogene in U2OS osteosarcoma cells. Azurin treatment may transform the function of p53 from inhibiting apoptosis to inducing apoptosis.

\section{Acknowledgements}

This study is supported by the National Nature Science Foundation of China (30672138), Nature Science Foundation of Zhejiang province in China (no. Y206257) and New Teacher Foundation of Ministry of Education in China. (20070335040).

\section{References}

1. Goto M, Yamada G, Kimbara K, Horner J, Newcomb M, Das Gupta TK and Chakrabarty AM: Induction of apoptosis in macrophages by Pseudomonas aeruginosa azurin: Tumour-suppressor protein p53 and reactive oxygen species, but not redox activity, as critical elements in cytotoxicity. Mol Microbiol 47: 549-559, 2003.

2. Yang DS, Miao XD, Ye ZM, Feng J, Xu RZ, Huang X and Ge FF: Bacterial redox protein azurin induce apoptosis in human osteosarcoma U2OS cells. Pharmacol Res 52: 413-421, 2005.

3. Ye Z, Peng H, Fang Y, Feng J and Yang DS: The construction of the eukaryotic expression plasmid pcDNA3.1/azurin and the increased apoptosis of U2OS cells transfected with it. Cell Mol Biol Lett: Apr 6, 2007 (E-pub ahead of print).

4. Apiyo D and Wittung-Stafshede P: Unique complex between bacterial azurin and tumor-suppressor protein p53. Biochem Biophys Res Commun 332: 965-968, 2005.

5. De Grandis V, Bizzarri AR and Cannistraro S: Docking study and free energy simulation of the complex between p53 DNA-binding domain and azurin. J Mol Recognit 20: 215-226, 2007.

6. Taranta M, Bizzarri AR and Cannistraro S: Probing the interaction between p53 and the bacterial protein azurin by single molecule force spectroscopy. J Mol Recognit 21: 63-70, 2008.

7. Bizzarri AR, Di Agostino S, Andolfi L and Cannistraro S: A combined atomic force microscopy imaging and docking study to investigate the complex between p53 DNA binding domain and azurin. J Mol Recognit 22: 506-515, 2009.

8. Taranta M, Bizzarri AR and Cannistraro S: Modelling the interaction between the N-terminal domain of the tumor suppressor p53 and azurin. J Mol Recognit 22: 215-222, 2009.

9 Yamaguchi $\mathrm{H}$, Bhalla $\mathrm{K}$ and Wang HG: Bax plays a pivotal role in thapsigargin-induced apoptosis of human colon cancer HCT116 cells by controlling Smac/Diablo and Omi/HtrA2 release from mitochondria. Cancer Res 63: 1483-1489, 2003.

10 Schlesinger PH, Gross A, Yin XM, Yamamoto K, Saito M, Waksman G and Korsmeyer SJ: Comparison of the ion channel characteristics of proapoptotic BAX and antiapoptotic BCL-2. Proc Natl Acad Sci USA 94: 11357-11362, 1997.

$11 \mathrm{Tu}$ Y, Xu FH, Liu J, Vescio R, Berenson J, Fady C and Lichtenstein A: Upregulated expression of BCL-2 in multiple myeloma cells induced by exposure to doxorubicin, etoposide, and hydrogen peroxide. Blood 88: 1805-1812, 1996.

12. Yamada T, Hiraoka Y, Ikehatat M, Kimbara K, Avner BS, Das Gupta TK and Chakrabarty AM: Apoptosis or growth arrest: Modulation of tumor suppressor p53's specificity by bacterial redox protein azurin. Proc Natl Acad Sci USA 101: 4770-4775, 2004. 